\title{
Oltre il sistema di accoglienza. La transizione abitativa dei rifugiati nella città di Milano
}

Citation: G. Gambazza, F. Lucchesi, M. Paradiso (2020) Oltre il sistema di accoglienza. La transizione abitativa dei rifugiati nella città di Milano. Bollettino della Società Geografica Italiana serie 14, 3(1): 81-92. doi: 10.36253/ bsgi.v3i1.1044

Copyright: @2020 G. Gambazza, F. Lucchesi, M. Paradiso. This is an open access, peer-reviewed article published by Firenze University Press (http:// www.fupress.com/bsgi) and distributed under the terms of the Creative Commons Attribution License, which permits unrestricted use, distribution, and reproduction in any medium, provided the original author and source are credited.

Data Availability Statement: All relevant data are within the paper and its Supporting Information files.

Competing Interests: The Author(s) declare(s) no conflict of interest.

For Italian evaluation purposes: Giuseppe Gambazza takes responsability for sections 1 and 2, Flavio Lucchesi for sections 3 and 4, Maria Paradiso for sections 5 and 6 .

\section{Beyond the reception system. Refugees' access to housing in the city of Milan. Abstract}

\author{
Giuseppe Gambazza, Flavio Lucchesi, Maria Paradiso \\ Dipartimento di Beni Culturali e Ambientali, Università degli Studi di Milano, Italia \\ E-mail: giuseppe.gambazza@unimi.it, flavio.lucchesi@unimi.it, maria.paradiso@unimi.it
}

\begin{abstract}
This study is anchored on the perspective of local turn in migrant integration, which requires cities to play an increasingly important role in defining public policies. Consequently, the research focuses on the explorative case of the city of Milan, aiming to demonstrate whether its local system provides a toolbox for refugees to promote their integration in the host/majority milieus. Through a qualitative method, based on semi-structured interviews with politicians, local officers, NGO activists, volunteers, and refugees, we will try to understand élite and non-élite perspective about how and where the refugees of the reception system of Milan can find an accommodation, at the end of the institutional process. In this way, it will also be possible to understand how the municipality is facing the restrictions to the Italian asylum system, the crisis of the welfare, the market-dominated systems of housing provision and the suspiciousness or racism towards immigrants, considered as a burden or a threat for the society.
\end{abstract}

Keywords: Integration, refugees, housing, Milan.

Riassunto. Lo studio si inquadra nella prospettiva del local turn, secondo la quale le città sono chiamate a svolgere un ruolo sempre più importante nella definizione delle politiche pubbliche nell'integrazione dei migranti. Si concentra, in particolare, sul caso della città di Milano, nel tentativo di dimostrare se il sistema locale di accoglienza fornisca ai rifugiati gli strumenti adeguati a integrarsi nella comunità ospitante. Utilizzando una metodologia qualitativa, basata su interviste semi-strutturate con funzionari locali, attivisti di ONG, membri del Terzo settore e volontari, si è tentato nello specifico di comprendere se e come i rifugiati del sistema di accoglienza di Milano possano trovare un alloggio, al termine del processo istituzionale. In tal modo, si è anche tentato di capire come il Comune stia affrontando le restrizioni al sistema di asilo italiano, la crisi del welfare, i sistemi di offerta di alloggi dominati dal mercato, il sentimento di sospetto o razzismo nei confronti degli immigrati, considerati come un peso o una minaccia per la società.

Parole chiave: Integrazione, rifugiati, Housing, Milano 


\section{Introduzione. Quale paradigma per l'accoglienza dei rifugiati?}

La gestione dei flussi migratori coinvolge oggi un gruppo eterogeneo di attori, impegnati nella costruzione di un nuovo sistema di governance, che ha ridefinito il tradizionale modello governativo, ridimensionando il ruolo dell'organo statale (Giudici, Wihtol de Wenden 2016).

Se in passato, infatti, le interazioni fra Stato e altri enti erano gerarchicamente strutturate (Gill 2010), le attuali policies dell'immigrazione sono spesso il risultato di rapporti (conflittuali o condivisi) tra attori le cui aree di influenza non appaiono chiaramente determinate.

$\mathrm{Ne}$ dipende una governance a geometria variabile, in cui competenze, poteri e spazi di manovra di ciascun partecipante non risultano essere preventivamente determinati, ma sono il frutto di una costante opera di ri-negoziazione.

Ė pur vero, però, che le autorità statali ricoprono ancora un ruolo preminente in molti settori dell'immigrazione, specialmente in quelli legati al controllo della mobilità e dei confini, benché anche in questi casi esse possano adeguare le proprie strategie sulla spinta di pressioni provenienti dal basso (Anderson 2016).

Altre volte lo stato-nazione è obbligato a cedere parte del proprio potere: ciò accade quando si trova impossibilitato ad affrontare autonomamente alcuni aspetti delle migrazioni globali e decide così di demandarne la risoluzione a organismi sovranazionali. Ne forniscono un esempio i programmi di resettlement dell'UE, che impegnano gli Stati membri ad accogliere quote di rifugiati, nel tentativo di redistribuire il "fardello" dei migranti in modo equo (Robinson et al. 2003).

È tuttavia rispetto alle pratiche dell'accoglienza che si esprime in modo più evidente il modello di governance di cui si è detto, caratterizzato dal progressivo coinvolgimento di attori locali. Se è vero che sono le autorità nazionali a stabilire il modello di integrazione adottato in un determinato Paese (che a seconda dei casi può assumere un carattere assimilazionista, multiculturalista o interculturale), sempre più spesso spetta alle autorità pubbliche, alle organizzazioni non governative, ai movimenti sociali (attivi "sul territorio") il compito di integrare le risoluzioni prese a livello centrale, nonché di ovviare alla loro inefficacia (Scholten, Penninx 2016).

Dal punto di vista epistemologico, i rivolgimenti sopra descritti hanno anche condotto a un importante cambio di paradigma, che è coinciso con la progressiva marginalizzazione del cosiddetto 'nazionalismo metodo- logico'1 e con lo sviluppo di nuovi approcci che individuano nelle caratteristiche socio-territoriali della scala locale i fattori determinanti nel processo di integrazione (Phillips, Robinson 2015; Glick-Schiller, Çağlar 2009).

Analizzare il livello di coinvolgimento dei diversi soggetti attivi nel sistema 'orizzontale' di accoglienza, le loro interrelazioni e l'eventuale inquadramento della governance locale in un sistema multilivello (ZapataBarreiro et al. 2017) permette infatti di scardinare la tradizionale visione delle politiche dell'immigrazione, fondata su una struttura predeterminata e gerarchicamente definita in cui l'autorità centrale ricopriva il ruolo determinante.

Gli studi "localisti" rivalutano il ruolo delle città nelle pratiche di integrazione. Le aree urbane, infatti, grazie alla loro propensione (presupposta o reale) nel creare legami, ospitare differenze e favorire incontri, possono costituire spazi privilegiati per ospitare e produrre buone pratiche di accoglienza (Caponio et al. 2010; 2019).

Esistono città che applicano le direttive centraliste in materia di accoglienza, spesso di natura securitaria (Harvey 1973), confinando i rifugiati in zone liminali, dove restano in attesa di vedersi riconosciuto il pieno diritto di accedere a beni e servizi (Agamben 2005; Darling 2013; Sanyal 2012; Kreichauf 2018). A monte di tali comportamenti ci sarebbe un eccessivo scarto tra il limitato ruolo decisionale riconosciuto all'ente locale nel costruire processi duraturi volti all'integrazione e la complessità delle problematiche affrontate (Glorious, Doomernik 2020).

Tuttavia, altri modelli di accoglienza urbana rifiutano il sistema centrale di accoglienza e operano nel tentativo di modificarlo o sovvertirlo: «la città può [dunque] diventare uno spazio per una politica di critica nei confronti dello Stato, una politica che rifiuta specifiche forme di governmentality, in particolare l'abiezione degli sfollati» (Darling 2017, 192). A seconda dei casi essa può sopperire alle mancanze dell'autorità centrale in materia di integrazione (Derrida 2011; Young 2011), ergersi come ultimo baluardo umanitario di fronte all'avanzata di un diffuso sentimento anti-immigrazione (Babels 2018) oppure organizzarsi in una rete finalizzata a esercitare pressione sugli organi decisori, come è accaduto con la formazione del network europeo delle 'città di rifugio', che stimola l'UE affinché essa adotti misure più attente $\mathrm{e}$ adeguate alle sfide cui far fronte (Eurocities 2015).

Altre volte, infine, le policies di accoglienza si inseriscono nell'ambito di accordi sottoscritti tra poteri locali

\footnotetext{
${ }^{1}$ Per una rassegna delle teorie che rigettano la visione del "nazionalismo metodologico", si veda Samers e Collyer (2017)
} 
e sovra-locali, che possono costituire forme di governance multilivello strutturate, finalizzate a sviluppare una cooperazione (verticale) continua e duratura con gli enti locali (Scholten 2019).

\section{Verso una governance dell'integrazione abitativa: aspetti teorici e metodologici di ricerca}

Un particolare aspetto dell'accoglienza dei rifugiati in città è quello legato all'inserimento abitativo.

Molto frequentato sia negli scritti scientifici (Alexander 2007; Ager, Strang 2008) sia nella reportistica internazionale di monitoraggio ${ }^{2}$, il tema della casa rappresenta un elemento fondamentale per misurare il livello di integrazione fra le comunità ospitanti e i nuovi arrivati (Netto 2011).

Nell'housing può infatti declinarsi localmente il concetto di "spazio di protezione", inteso come «un ambiente favorevole al godimento dei diritti dei rifugiati riconosciuti a livello internazionale» (UNHCR 2009, 4), in cui i rifugiati possono accedere con maggiore o minore facilità ad altri servizi fondamentali.

Vivere in alloggi collettivi rende difficile l'apprendimento (...), l'essere ospitati in centri di accoglienza remoti senza mezzi pubblici complica la vita quotidiana e l'accesso al mercato del lavoro (...). Inoltre, il tipo e le dimensioni dell'abitazione influiscono sulla percezione che la società ha dei rifugiati. Per esempio, la creazione di alloggi collettivi favorisce la diffusione tra gli abitanti locali di atteggiamenti negativi nei confronti dei rifugiati e (...) spesso porta a conflitti tra l'amministrazione e la popolazione locale (Adam et al. 2020, 206).

Da un punto di vista concettuale, il tema dell'integrazione abitativa coinvolge complesse dimensioni socioeconomiche, giuridiche, politiche e culturali (Castles et al. 2002). Tale complessità ha pertanto favorito la nascita di differenti correnti di pensiero, che individuano diverse prospettive di indagine, volte a studiare il fenomeno secondo un'ottica strutturalista o socioculturale.

L'impostazione "strutturalista" ha dominato l'analisi della situazione abitativa dei gruppi etnici minoritari, indagando l'accesso ad alloggi di buona qualità e a prezzi accessibili, la soddisfazione dei bisogni abitativi e la gestione del processo di insediamento a livello di comunità locale (Phillips 2006). Tale prospettiva si concentra soprattutto sui fattori che rendono difficile l'accesso all'alloggio o limitano la scelta nel processo di ricerca.

\footnotetext{
2 Si vedano l'indice MIPEX (Migrant Integration Policy Index) (https://
} mipex.eu) e quello realizzato da Wolffhardt et al. (2019).
D’altro lato, si sono diffusi approcci maggiormente interessati allo studio dei percorsi alloggiativi, più attenti a definire i ruoli degli attori coinvolti nel processo insediativo e le relative interrelazioni. Tali analisi hanno ridefinito la figura stessa del rifugiato, non più considerato come soggetto passivo, mosso da forze esterne, ma come parte di una rete territoriale in grado di sviluppare strategie per superare le barriere talvolta stabilite dalla società dominante. Valgano gli esempi degli studi relativi all'housing pathway (Clapham 2002) o che indagano l'integrazione alla luce del concetto di capitale sociale (Putnam 2000), secondo cui le scelte abitative sarebbero meglio comprensibili analizzando i legami sociali che i rifugiati intessono con la famiglia e i gruppi co-etnici (bonding) e/o con membri della società ospitante (bridging).

Gli approcci socioculturali rappresentano validi strumenti per cogliere la complessità delle dinamiche abitative di rifugiati e richiedenti asilo nei primi anni dopo l'arrivo, mostrando come il reperimento di un alloggio sia correlato al tipo di relazioni tra gli attori coinvolti nel settore abitativo (come amici, parenti, agenti immobiliari, avvocati, proprietari, assistenti sociali e funzionari pubblici), la loro posizione sociale e le loro risorse disponibili e realizzabili (Aigner 2019).

Intersecando l'approccio socioculturale dell'integrazione abitativa con le teorie del local turn, questa ricerca affronta il modo in cui gli attori della società ospitante si organizzano localmente per dare supporto ai rifugiati in uscita dai percorsi istituzionali di seconda accoglienza, valorizzando aspetti sociali, culturali ed emozionali (Berry 2006; Diab et al. 2019), volti a favorire l'integrazione abitativa.

Fatte salve alcune eccezioni (cfr. Ferrario 2019), si ritiene infatti che non sia stata finora riservata sufficiente attenzione al modo in cui gli attori della società ospitante si organizzino localmente per dare supporto ai rifugiati in uscita dal sistema SIPROIMI (Sistema di protezione per titolari di protezione internazionale e per i minori stranieri non accompagnati) i quali, per soddisfare il proprio bisogno abitativo, devono misurarsi con una realtà meno normata, caratterizzata da una crisi generale del welfare (Andreotti, Mingione 2014) e prevalentemente determinata dalle leggi di mercato (Allen et al. 2004).

Prendendo in esame il sistema di seconda accoglienza nella città di Milano - centro di immigrazione tra i principali d'Italia (Cristaldi 2012) e "modello" per l'accoglienza dei richiedenti asilo e rifugiati (Bini, Gambazza 2019) - questo studio mira ad analizzare le caratteristiche della rete impegnata a offrire un supporto ai rifugiati, nella ricerca di alloggi dignitosi e sicuri durante la fase di transizione abitativa. 
Si valuterà poi in che modo le policies urbane legate all'housing dei rifugiati declinino localmente le direttive nazionali sull'accoglienza. Più precisamente si tenterà di rispondere alle seguenti domande di ricerca:

- Quali sono le opportunità abitative per i beneficiari in uscita dal SIPROIMI?

- Quali attori esercitano un ruolo attivo nel favorire l'inserimento abitativo dei rifugiati in uscita dal SIPROIMI? Quali sono meno partecipi?

- Esistono collaborazioni fra i vari attori coinvolti nel processo (governo centrale, autorità locali, attori privati, cittadinanza)?

Muovendo da tali quesiti si intende delineare il modello milanese di inserimento abitativo per i rifugiati, individuandone potenzialità e limiti, anche al fine di immaginarne un valido percorso di sviluppo.

Per raggiungere gli obiettivi della ricerca, è stato impostato uno studio qualitativo, basato sull'analisi dei documenti ufficiali e sulla somministrazione di 14 interviste semi-strutturate (della durata media di 90 minuti) a testimoni privilegiati, individuati attraverso un campionamento a valanga e scelti tra i seguenti gruppi di attori:

i. membri di associazioni e ONG impegnate all'integrazione dei rifugiati (4 persone),

ii. dipendenti della Pubblica amministrazione (3 persone),

iii.operatori dei Centri di accoglienza SIPROIMI (5 persone),

iv. professionisti dell'housing (2 persone).

Ogni intervista è composta complessivamente da 12 domande e suddivisibile in due parti, ciascuna delle quali mira ad approfondire un particolare aspetto della ricerca.

La prima parte si concentra su opportunità e ostacoli che i rifugiati incontrano durante la fase di transizione abitativa e risulta così organizzata:

- Come giudica il sistema attuale in merito all'alloggio per le persone fragili?

- Di quali strumenti i rifugiati in uscita dal SIPROIMI dispongono per trovare un alloggio?

- Quali sono le opportunità abitative per i beneficiari in uscita dal SIPROIMI?

- Esiste un sostegno informale per l'ingresso nel mercato immobiliare?

- Quali sono i maggiori impedimenti che i rifugiati incontrano nell'ingresso nel mercato immobiliare?

- Ci sono gruppi di rifugiati che hanno maggiori facilità a inserirsi? Per quali motivi?

- Quali gruppi hanno più difficoltà e perché?

La seconda parte dell'intervista si concentra mag- giormente su aspetti legati alla governance della fase di transizione abitativa, che vengono indagati attraverso la somministrazione delle seguenti domande:

- Come valuta l'operato dell'ente pubblico?

- Come giudica quello del Terzo settore?

- E l'impegno dei cittadini?

- Ci sono enti con cui collaborate in modo privilegiato?

- Come pensa che la situazione potrebbe essere migliorata?

L'approfondimento del campo di indagine è avvenuto inoltre con la partecipazione al panel 'La questione abitativa per i titolari di protezione internazionale in uscita dal SIPROIMI' del progetto internazionale National Integration Evaluation Mechanism (NIEM PROJECT) $)^{3}$. Il lavoro sul campo è stato condotto tra ottobre 2019 e gennaio 2020.

\section{Oltre la "seconda accoglienza". L'inserimento abitativo ai tempi del Decreto Sicurezza}

Nel 2018 le richieste di asilo registrate in Italia sono state circa $53.596^{4}$, di cui la maggior parte presentate da persone provenienti da Pakistan (7.368 domande), Nigeria (6.336), Bangladesh (5.026), Senegal (2.867) e Ucraina (2.517).

$\mathrm{Nel}$ corso dello stesso anno le 20 Commissioni territoriali, preposte al riconoscimento della protezione internazionale, hanno preso in esame 95.576 richieste di asilo, accogliendone soltanto una parte esigua. A beneficiare dello status di rifugiato sono stati infatti 7.096 richiedenti (circa il 7\% del totale), mentre altre forme di protezione sussidiaria e umanitaria ${ }^{5}$ sono state accordate rispettivamente a $4.319(5 \%)$ e $20.014(21 \%)$ persone $^{6}$.

${ }^{3}$ Il progetto NIEM è finalizzato all'analisi delle normative, delle politiche e delle prassi implementate in 15 Paesi membri dell'UE, a supporto dell'integrazione dei beneficiari di protezione internazionale. Il suddetto tavolo di lavoro sull'housing dei rifugiati si è riunito il giorno 13 dicembre 2019 presso la sede della Fondazione Ismu (Initiatives and Studies on Multi-ethnicity), che è partner del progetto.

${ }^{4}$ L'Italia risulta essere il quinto Paese UE per richieste di asilo, alle spalle di Germania, Francia, Grecia e Spagna (https://ec.europa. eu/eurostat/statistics-explained/index.php?title=Asylum_statistics/ it\&oldid=449355\#Diminuzione_del_numero_di_richiedenti_asilo_ nel_2018).

${ }^{5}$ La L. 132/2018 (il cosiddetto Decreto Sicurezza) ha sancito l'abolizione dei "motivi umanitari" tra le concessioni di permesso di soggiorno per i richiedenti asilo. Una misura particolarmente contestata, perché rischia di creare nuove sacche di marginalità sociale, anche rafforzando il lavoro nero e la piccola criminalità (ASGI, 2018: https://www.asgi.it/ wp-content/uploads/2018/10/ASGI_DL_113_15102018_manifestioni_ illegittimita_costituzione.pdf).

${ }^{6}$ I dati relativi alle richieste delle varie forme di protezione e ai relativi esiti sono stati elaborati dagli Autori sulla base delle evidenze fornite dal Dipartimento per le libertà civili e l'immigrazione - Ministero dell'Inter- 
Tali evidenze mostrano come quello dell'accoglienza rappresenti da anni un tema rilevante nel dibattito nazionale ${ }^{7}$. In tempi recenti, infatti, più volte il sistema di accoglienza è stato ripensato nel tentativo (reale o presupposto) di far fronte prontamente al succedersi delle diverse ondate migratorie che hanno interessato il Paese. In tale solco si inserisce la L. 132/2018 (il cosiddetto "Decreto Sicurezza"), da cui sono dipese le più recenti e significative trasformazioni riguardanti le attività di prima e seconda accoglienza, rispettivamente rivolte ai richiedenti asilo e ai beneficiari di protezione internazionale.

La prima fase dell'accoglienza è gestita prevalentemente a livello governativo, attraverso una rete di strutture deputate a ospitare i richiedenti asilo durante l'espletamento delle operazioni atte a definirne la posizione giuridica. A loro volta le strutture di prima accoglienza si suddividono fra hotspots (90 ospiti), CPA (Centri governativi di Prima Accoglienza di cui all'articolo 9 del D. Lgs. 142/2015) e CAS (Centri di Accoglienza Straordinaria di cui all'articolo 11 del D. Lgs. 142/2015), che complessivamente accolgono 87.723 ospiti $^{8}$.

La seconda fase, denominata SIPROIMI (30.682 posti $\left.i^{9}\right)$, è invece erogata grazie alla collaborazione tra il Comune e gli enti del Terzo settore ed esclusivamente rivolta ai beneficiari di varie forme di protezione $e^{10}$.

L'accesso al SIPROIMI è la sola condizione per essere inseriti in percorsi strutturati di integrazione della durata standard di 6 mesi (con possibilità di proroghe multiple), finalizzati a offrire gli strumenti necessari per l'apprendimento della lingua italiana, l'orientamento legale, l'assistenza psicologica, la tutela della vita familiare (EMN 2018).

Per quanto riguarda l'inserimento abitativo dei rifugiati in uscita dal sistema di accoglienza, si riscontra tuttavia un eccessivo scollamento tra la situazione auspicata e quella reale. Se, da un lato, il "Piano nazionale d'integrazione dei titolari di protezione internazionale" $(2017)^{11}$ considera l'alloggio come parte rilevante dei

no: http://www.libertaciviliimmigrazione.dlci.interno.gov.it/sites/default/ files/allegati/riepilogo_anno_2018.pdf.

${ }^{7}$ Un parziale (e inevitabilmente lacunoso) elenco di rilevanti scritti, che partecipano alla vivace e proficua riflessione sull'argomento, comprende: Amato 2008; Ambrosini 2010; Brusa 2012; Cassi, Meini 2002; Cristaldi 2019; Gavinelli 2013; ISMU 2019; Krasna 2009; Meini e Salvatori 2018; Terranova 2017.

${ }^{8}$ Cfr. https://www.camera.it/application/xmanager/projects/leg18/ attachments/upload_file_doc_acquisiti/pdfs/000/001/791/Memorie_Prefetto_Michele_Di_Bari.pdf.

${ }^{9} \mathrm{Cfr}$. https://www.siproimi.it/i-numeri-dello-sprar.

${ }^{10}$ Per approfondimenti si vedano Aru 2019; Omenetto 2019; Semprebon, Pelacani 2020.

${ }^{11} \mathrm{Cfr}$. https://www.interno.gov.it/sites/default/files/piano_nazionale_ integrazione.pdf percorsi di inclusione sociale, dall'altro esso non prevede uno stanziamento di risorse ad hoc. Di conseguenza, il sistema nazionale di seconda accoglienza si concentra in modo marginale sull'inserimento abitativo, come dimostra il fatto che, nel 2017, i progetti SPRAR destinati all'housing hanno rappresentato il 5\% del totale dei servizi erogati (Ministero dell'Interno 2018).

Un secondo impedimento all'integrazione abitativa sarebbe rappresentato dalla diffidenza mostrata dalle agenzie immobiliari e dai proprietari degli immobili verso i rifugiati. I proprietari sono infatti spesso restii a sottoscrivere un contratto di locazione a un rifugiato, la cui presenza - in un clima di deriva populista (Gidron, Hall 2019) - viene considerata come minacciosa (Collyer, King 2015) e rischierebbe di abbattere il valore degli immobili.

Ulteriori ostacoli hanno riguardato, inoltre, la situazione di precarietà lavorativa dei beneficiari di protezione internazionale e l'assenza di forme di sostegno attivate anche da reti sociali infra-etniche: abbandonati a se stessi, i nuovi arrivati rischiano infatti di non venire a conoscenza delle opportunità a loro riservate dalla società ospitante.

A determinare lo svantaggio abitativo contribuiscono inoltre elementi di natura strutturale, legati alle normative e alle condizioni del mercato immobiliare.

Un primo ordine di difficoltà deriva dal fatto che il diritto alla casa - richiamato nell'art. 47 della Costituzione e in ripetute sentenze della Consulta ${ }^{12}$ - risulta essere difficilmente esigibile e impugnabile in sede giudiziale, oltreché applicabile in modo disarmonico nei diversi territori regionali. Né si prevedono particolari tutele per i titolari di protezione internazionale i quali, una volta dimessi dai percorsi di seconda accoglienza, sono sottoposti alle medesime condizioni dei cittadini italiani, per quanto concerne le forme di sostegno per l'accesso agli alloggi pubblici, al mercato della locazione privata o all'acquisto della prima casa.

La preminenza del settore privato e delle logiche di mercato nel sistema di fornitura di alloggi (Allen et al. 2004) - resi particolarmente evidenti dalla liberalizzazione dei canoni di affitto (regolamentata principalmente dalla "Legge Zagatti" 431/1998) - ha determinato inoltre l'innalzamento dei costi di locazione, divenuti proibitivi per le persone più povere, e intensificato la corsa all'acquisto della casa di proprietà (Caruso 2017; Pittini et al. 2017). Parimenti si è verificato il progressivo trasferimento alle Regioni e agli enti locali delle competen-

\footnotetext{
${ }^{12} \mathrm{Si}$ fa qui riferimento, ad esempio, alla L. 49/1987; Corte Cost. sent. n. 217 del 1988; Corte Cost. sent. n. 119 del 24 marzo 1999; Corte Cost. sent. n. 217 del 25 febbraio 1988; Corte Cost. sent. n. 252 del 1983.
} 
ze relative all'abitare ${ }^{13}$, che in alcuni casi hanno stabilito restrizioni circa l'assegnazione di alloggi di edilizia popolare, con l'esclusione di particolari gruppi di persone, come i rifugiati.

$\mathrm{Va}$ infine ricordato che la contrazione dell'edilizia pubblica, solo parzialmente contrastata dalle recenti misure che mirano a coinvolgere gli attori privati, non riguarda unicamente la popolazione immigrata, ma coinvolge strati sempre più ampi della società: 1'88\% delle famiglie presenti in alloggi di edilizia popolare è infatti di origine italiana (Agustoni 2018). Tale situazione inasprisce le condizioni dei rifugiati, i quali si trovano sottoposti a una maggiore concorrenza per l'accesso a un alloggio.

\section{L'housing dei rifugiati a Milano: un'analisi di scenario}

A fornire uno spaccato delle attuali condizioni del sistema di seconda accoglienza milanese è il "IX Rapporto statistico sui rifugiati e i Richiedenti Asilo a Milano", commissionato dall'Unità politiche per l'inclusione e l'immigrazione del Comune di Milano (Comune di Milano 2018).

Tale indagine mostra come a Milano nel 2018 il sistema SIPROIMI rendesse disponibili 422 posti.

L'offerta erogata grazie alla collaborazione tra il Comune e gli enti del Terzo settore (Casa della Carità, Comunità Progetto, Consorzio Farsi Prossimo, Cooperativa Farsi prossimo, Fondazione Progetto Arca, La Grangia Monluè) consta di 7 centri di accoglienza che accolgono 1'80\% degli ospiti totali e di una rete di appartamenti dislocati sul territorio.

Tale rete di strutture ha dovuto modificarsi per far fronte al progressivo aumento degli arrivi annui, che dal 2011 al 2017 sono passati da 89 a 415, con un incremento del 366\%, interrotto soltanto nel 2018 (396 ingressi).

Nonostante la riduzione degli sbarchi e, di conseguenza, degli arrivi in Italia di richiedenti asilo, la domanda di posti di seconda accoglienza è rimasta pressoché invariata nel corso del 2018.

Osservando le caratteristiche socioculturali degli ospiti del sistema di seconda accoglienza milanese, si evince che il $60 \%$ del totale degli ospiti è rappresentato da migranti provenienti dall'Africa, in particolare somali. Prosegue, invece, il decremento di afghani e pakistani, i cui ingressi sono circa dimezzati rispetto all'anno

\footnotetext{
${ }^{13}$ Con tale disposizione, perfezionata dalla riforma del titolo V della Costituzione (Legge n. 3/2001) e dalla sentenza della Corte Cost. sent. n. 94/2007, le Regioni si trovano in particolare a gestire le agenzie locali che sostituiscono i dismessi Istituti Autonomi Case Popolari (IACP).
}

precedente.

Le piramidi delle età si mantengono tendenzialmente inalterate nel periodo considerato e individuano una popolazione composta prevalentemente da maschi $(83 \%)$ e tendenzialmente giovane: il $90 \%$ dei beneficiari sono di età inferiore ai 34 anni, con una quota di minori pari a circa il $6 \%$.

A far registrare i più sensibili cambiamenti rispetto al passato è la composizione degli utenti con riferimento allo status giuridico. Nel 2018 la quota di richiedenti asilo (7\%) presenti nelle strutture risulta ai minimi storici, mentre appare in sensibile aumento la percentuale dei beneficiari di protezione internazionale (37\%): la progressiva esclusione dai SIPROIMI dei richiedenti asilo, esito del Decreto sicurezza, sarà presumibilmente confermata anche negli anni a venire.

Sebbene il periodo previsto per il funzionamento del servizio sia di 6 mesi, quasi la metà dei soggetti usufruisce dell'accoglienza per un periodo inferiore ai tempi standard: spesso la causa della dimissione è dovuta a scelte volontarie (30\% dei casi) del singolo utente che decide di anticipare la naturale conclusione del periodo di accoglienza, talvolta senza informare gli operatori. Tali “dimissioni per irreperibilità" risultano più frequenti fra le donne $(32,7 \%$ del totale) e fra i membri dell'etnia somala $(22,8 \%)$.

Appare più complesso stabilire la soluzione abitativa adottata dall'utente dimesso. Dell'ampia maggioranza degli utenti dimessi dal SIPROIMI milanese nell'anno 2018 (325 su 386) non è nota la destinazione.

Alcune cause di tale dispersione vanno ricercate nel modello di welfare abitativo previsto a livello regionale, che ha subito importanti modifiche volte a rispondere alle esigenze abitative di una sempre più ampia fetta di popolazione.

Con la Legge Regionale 16/2016 (e i successivi emendamenti), la Regione Lombardia ha reso difficoltoso per i rifugiati concorrere per un alloggio di edilizia a canone calmierato. Le graduatorie favoriscono, infatti, chi è residente sul territorio da almeno 5 anni, penalizzando coloro che non presentano uno status giuridico idoneo.

Inoltre, per rendere il settore dell'edilizia residenziale economicamente sostenibile ${ }^{14}$, la normativa ha fissato un tetto ai posti riservati alle persone più fragili, che si attestano al $20 \%$ del totale, a tutto vantaggio di altre dalle maggiori capacità economiche.

A Milano, in particolare, l'edilizia pubblica rappre-

\footnotetext{
14 Tale tendenza si è verificata in seguito al crescente coinvolgimento dell'attore privato e alla sperimentazione di 18 progetti di housing sociale attivi in Lombardia, che hanno predisposto 1938 alloggi e 278 posti letto. Di essi però solamente l'11\% è a canone sociale, cioè rivolto a famiglie con un ISEE-ERP inferiore a 16.000 euro (Puja 2018).
} 
senta solo l'11\% del settore immobiliare ed è composta da abitazioni di proprietà dell'Azienda Lombarda per l'Edilizia Residenziale (ALER) o del Comune di Milano. In generale lo stock di edilizia pubblica appare piuttosto limitato rispetto al numero potenziale di persone che ne avrebbero diritto, anche in considerazione della presenza di un mercato degli affitti non regolamentato (Cittalia 2010; Bricocoli, Cucca 2016).

Nel capoluogo lombardo, infatti, nel 2018 sono pervenute 25.000 richieste di alloggio in edilizia residenziale pubblica, a fronte dei soli 859 alloggi disponibili.

Tale emergenza abitativa ha condotto il capoluogo lombardo a mettere in atto alcune misure specifiche, che vanno dall'individuazione di ulteriori alloggi di edilizia residenziale pubblica, assegnati in deroga alla graduatoria regionale (www.comunemilano.it), all'ospitalità in hotel a 25 famiglie in difficoltà, fino all'implementazione del programma di Residenzialità Sociale Temporanea (RST), che mette a disposizione 500 unità abitative in condivisione.

A tali interventi si affiancano altre iniziative "orientate a dare risposte innovative ai bisogni emergenziali", che garantirebbero ulteriori 160 alloggi ${ }^{15}$.

\section{Le interviste in profondità}

Le interviste somministrate agli operatori attivi sul territorio milanese consentono di definire con maggiore chiarezza il quadro sopra esposto, mettendone particolarmente in risalto le criticità e gli aspetti positivi, oltre a ipotizzarne alcune auspicabili trasformazioni.

Le testimonianze raccolte confermano le difficoltà già individuate nell'analisi documentale nel reperire informazioni esaustive riguardanti la sistemazione abitativa dei rifugiati in uscita dal SIPROIMI:

Non sappiamo dire con certezza dove vadano i rifugiati una volta usciti da qui: molti non te lo dicono, non vogliono, non se la sentono, magari si vergognano. Qualcuno dice di rimanere a vivere a Milano e dopo 3-4 mesi scopri da alcuni suoi connazionali che si è trasferito in Svezia. C'è una "barriera", non saprei definirla altrimenti, che li separa persino da noi, che ci passiamo insieme 6, 9, 12 mesi. Ma non possiamo essere sicuri che ci dicano la verità: anche chiedendo loro espressamente dove andranno ad abitare, non è detto che tutti ti daranno risposta: qualcuno lo farà e qualcuno no (Int. 1, Mattia, operatore SIPROIMI).

Più nello specifico, gli intervistati concordano nel sottolineare l'esistenza di un problema specifico lega-

\footnotetext{
${ }^{15}$ Per approfondimenti, si rimanda a Naga (2019).
}

to alla scarsità di posti e di possibilità alloggiative. Le soluzioni adottate sono dunque le più varie, a partire dall'ingresso in dormitori pubblici (come i locali del Piano freddo, il dormitorio 'Casa Jannacci', la 'Casa della Carità'), predisposti ad accogliere le persone più fragili, a cui si rivolgono coloro che escono dalla seconda accoglienza senza aver raggiunto gli strumenti adatti all'integrazione. Altri decidono di appoggiarsi alla rete dei loro connazionali, con i quali condividere un appartamento, così da ridurre, per altro, le spese di affitto. Una fetta considerevole decide invece di lasciare l'Italia, per ricongiungersi con i propri affetti residenti in altri Paesi europei.

Esistono, infine, casi eccezionali, rappresentati da coloro che hanno disponibilità economiche sopra la media e possono permettersi un alloggio di maggiore qualità, oppure da chi può usufruire di un ulteriore periodo di integrazione, per aver seguito un percorso particolarmente virtuoso o perché presenta specifiche fragilità.

In assenza di programmi istituzionali di inserimento, a determinare l'eventuale buona riuscita di ciascun insediamento concorre, dunque, una molteplicità di elementi, che abbracciano fattori legati al capitale sociale, culturale, economico, emozionale, che un individuo si è costruito nell'arco della sua esperienza di vita. A tal proposito, maggiori capacità adattive sono riconosciute ai giovani maschi (di circa trent'anni di età), mentre le famiglie con figli piccoli incontrerebbero le maggiori difficoltà:

Per le famiglie è molto difficile perché giustamente non si adattano a soluzioni che potrebbero essere non adeguate al bambino, alla moglie ecc. Gli uomini single di circa 30 anni di età si adattano, invece, molto più facilmente anche a situazioni estreme, anche rispetto ad anziani (Int. 2, Martina, operatrice SIPROIMI).

Tuttavia, la sistemazione ideale, culminante con la stipula di un regolare contratto di locazione, è riscontrabile solo in casi isolati. Più frequente è il fallimento dei percorsi di integrazione abitativa, come dimostrano diffusi fenomeni di abusivismo e di occupazione illecita di alcuni stabili.

Moltissime persone vivono in strada. Dormono in capanne nel parco, in vecchi capannoni abbandonati, in fabbriche dismesse e magari pericolanti, a volte completamente sprovviste di servizi igienici (Int. 4, Emilia, membro di associazione di volontariato).

Tra i fattori ostativi che i rifugiati incontrano nella ricerca di un alloggio decoroso, sicuro e abbordabile, si 
segnala il ruolo controverso adottato dalle istituzioni, accusate di assumere, di volta in volta, misure "inefficaci", "ostative" o "ambigue". Vengono in particolare addossate all'organo centrale le responsabilità di non aver regolamentato questa delicata fase di transizione.

Non c'è una visione complessiva, non c'è ufficio, un posto dove i rifugiati possano rivolgersi. Il problema è che manca una legge, lo Stato se ne frega. Tutto sta alla autonomia raggiunta dalle singole persone. (Int. 5, Mimma, membro di associazione di volontariato).

Un ulteriore effetto negativo è da ascrivere al Decreto Sicurezza, che ha abolito i percorsi di integrazione durante la fase di prima accoglienza, confinando migliaia di richiedenti asilo in un "limbo" spazio-temporale dalla durata indeterminata, e ha ridotto le risorse destinate all'accompagnamento dei rifugiati nell'uscita dai percorsi istituzionali.

Il servizio di accompagnamento delle persone dalla fuoriuscita fino al raggiungimento di una autonomia abitativa era un percorso in qualche modo strutturato. C'erano operatori dedicati che accompagnavano le persone a cercare l'appartamento, le mettevano in contatto con associazioni che disponevano di case a prezzo agevolato, facevano in modo che negli ultimi 6 mesi accantonassero soldi per poter pagare la caparra, i primi affitti, le prime utenze, le aiutavano a stendere il contratto. L'ente gestore stanziava inoltre dei fondi che avrebbero costituito il "primo gettone" di 500 euro per pagare il primo mese d'affitto. Era anche una tutela per il proprietario. Ora tutto dipende dalla discrezionalità di un determinato ente gestore, dalla sua etica professionale. (Int. 6, Guido, membro di associazione di volontariato).

Altre responsabilità sono imputate alla Regione, responsabile dell'aver promosso un sistema di edilizia pubblica e privata, particolarmente penalizzante per $\mathrm{i}$ rifugiati.

Del tema della residenza, la Lombardia ne ha fatto una bandiera ideologica: le case popolari agli italiani! (Int. 7, Valentina, professionista dell'housing).

L'ente comunale viene invece accusato di aver assunto una posizione equivoca rispetto al proble$\mathrm{ma}$, anche se ritenuto più attivo e partecipe di Stato e Regione nel confronto con le associazioni presenti sul territorio.
Sebbene abbia attivato iniziative specifiche volte alla risoluzione o alla limitazione del problema abitativo ${ }^{16}$, esso è risultato manchevole, secondo alcuni intervistati, nel processo di costruzione di una governance dell'accoglienza "orizzontale", spesso delegando indebitamente compiti agli attori del Terzo settore e del volontariato.

Spesso accade che quando un rifugiato sta cercando un posto dove andare a dormire, si rivolge al Centro Aiuto della Stazione Centrale (CASC). E il CASC non solo, nella maggioranza dei casi, non gli fornisce un alloggio... ma in piu ha l'ardire di mandarlo da noi... trasmettendogli un'informazione sbagliata.

Ciò è un'aberrazione politica: gli sportelli comunali indirizzano una persona a un'associazione, la quale deve necessariamente reindirizzarla al servizio pubblico (Int. 4, Emilia, membro di associazione di volontariato).

Le incoerenze del settore pubblico costringono $i$ rifugiati a misurarsi con il 'mercato', sprovvisti degli strumenti adatti: di fronte alla concorrenza di altre popolazioni fragili, al pregiudizio di proprietari e agenzie immobiliari, essi devono accettare sistemazioni economicamente svantaggiose, spesso raggiunte grazie a un “garante" autoctono, che funge da 'intermediario' con il proprietario. Più in generale, sul ruolo dei privati c'è discordanza di vedute: a fronte di chi ne critica fortemente l'operato, alcuni le considerano come un possibile fattore di miglioramento, auspicandone un maggiore coinvolgimento nell'integrazione abitativa.

Pareri complessivamente positivi vengono riservati al ruolo del volontariato milanese e del Terzo settore, sia di matrice religiosa sia laica, complessivamente costituito da attori propositivi e impegnati nel presidio di tale fase di transizione. Una particolare menzione meritano gli enti gestori delle strutture SIPROIMI, i cui operatori sono spesso coinvolti nell'attivazione di percorsi virtuosi in riferimento a un post-accoglienza più "strutturato".

La Diaconia Valdese mette a disposizione un operatore ogni 15 beneficiari e individua una figura interna specializzata nella formazione, che aiuta i rifugiati nella stesura dei loro curriculum vitae e li prepara ai colloqui di lavoro. Viene svolta una ricerca a tappeto sui bandi relativi alle questioni abitative e lavorative. Attualmente si sta cercando di costruire un progetto-ponte per il dopo: è in corso una trattativa con un'agenzia per delle case: si tratterebbe di un progetto di housing per un tempo determinato (Int. 8, Ilaria, operatrice SIPROIMI).

\footnotetext{
${ }^{16}$ Impiegati dellente locale hanno ricordato, a tal proposito, gli interventi specifici promossi dal Comune di Milano, di cui si è parlato nel capitolo 4 (Int. 10, Antonella, dipendente della Pubblica amministrazione).
} 
Voci critiche rimandano, ancora una volta, alla mancanza di coordinamento "orizzontale" fra i diversi enti. Se viene riconosciuto al Terzo settore un ruolo positivo nel percorso di accoglienza e integrazione abitativa, spesso gli viene imputata una leggera manchevolezza nel promuovere efficaci e chiare strategie di comunicazione. Capita infatti che un ente territoriale ignori policies, strumenti e risorse adottati da altre organizzazioni, precludendosi la possibilità di attivare collaborazioni con esse.

Non tutti gli enti territoriali sembrano sempre in contatto fra loro. Per esperienza, posso affermare che alcune associazioni lo siano, sebbene non ufficialmente, altre sono meno coinvolte (Int. 9, Marzia, membro di associazione di volontariato).

\section{Conclusioni}

La ricerca ha individuato la fase di transizione abitativa come il limbo spazio-temporale in cui i rifugiati in uscita dai percorsi istituzionali di seconda accoglienza ricercano una nuova sistemazione insediativa.

Nel dettaglio, la prima fase di studio, riguardante l'analisi delle opportunità abitative degli ex utenti del SIPROIMI, ha svelato una realtà dai contorni incerti e sfumati rispetto alla quale nemmeno gli addetti ai lavori hanno sempre saputo fornire esaustive spiegazioni e precise informazioni in merito: non è stato possibile, infatti, ricavare dati certi sulla localizzazione degli ex utenti del sistema di seconda accoglienza (2018). Né l'analisi documentale né le conversazioni (formali e informali) con addetti ai lavori hanno fornito evidenze certe in merito, a conferma del fatto che nella fase postaccoglienza si sviluppano dinamiche socio-territoriali 'opache', certamente meritevoli di ulteriori approfondimenti futuri, tuttavia determinate anche dalle incongruenze di policy.

A creare, difatti, incertezza concorrono la mancanza di una normativa specifica dedicata alla transizione abitativa e i tagli operati del Decreto Sicurezza nonché le culture politiche di tipo sovranista e populista che hanno riformato le leggi dello Stato, limitando sia l'azione statale sia le pratiche di accoglienza e integrazione. Di conseguenza, i rifugiati si trovano impreparati di fronte a un sistema di housing altamente concorrenziale, sempre più dominato dalle logiche di mercato e con la strada quasi sbarrata nelle graduatorie di edilizia popolare.

La seconda linea di ricerca, finalizzata a indagare il ruolo dei vari attori coinvolti durante la fase di transizione abitativa, ha individuato il ruolo decisivo delle azioni spontanee, provenienti dal basso, nell'ovviare alle falle del sistema istituzionale sia centrale che locale.

In uno scenario in cui il ruolo dell'autorità centrale risulta marginale e nel Parlamento si sono sedimentate culture con tratti a volte demagogicamente xenofobi, gli attori locali sono chiamati a svolgere una importante funzione ma in un contesto mediatico e social non favorevole. Tale funzione di governo locale, a volte, assume toni paradossali di critica verso l'azione legislativa statale che è stata, tuttavia, negli anni alimentata da partiti politici spesso di provenienza proprio lombarda e veneta.

La seconda linea di ricerca nell'analisi della realtà milanese ha mostrato, peraltro, come enti del volontariato e del Terzo settore contribuiscano a corroborare una rete di relazioni a cui il rifugiato - soggetto consapevole e proattivo, in grado di operare scelte individuali - possa rivolgersi per affrontare una particolare fase del suo percorso di immigrazione. Il capitale sociale, culturale, economico ed emozionale di cui sono dotati i rifugiati rappresenta, infatti, un'imprescindibile risorsa per chi si muove in un vuoto normativo e "affettivo" (Putnam 2000; Diab et al. 2019). Per questa ragione, coloro che escono dai percorsi di seconda accoglienza ricercano sostegno e appoggio all'interno di reti relazionali inter- e infra-etniche, le quali rappresentano spesso un baluardo di valore inestimabile.

In conclusione, con il tramonto delle politiche dell'accoglienza Stato-centriche non è ancora sorta un'adeguata governance multilivello, né un'adeguata cultura politica che si assuma le responsabilità del caso, non ultima quella di evitare rimostranze verso un Parlamento e una filiera istituzionale locale-centrale nella quale tutto sommato leader e forze locali rimangono protagonisti.

Tali lacune sono soltanto in parte ovviate dagli enti locali che mettono in atto iniziative meritorie, ma parzialmente efficaci e non perfettamente coordinate.

Senza un ripensamento generale del sistema di accoglienza, nel quale siano meglio definite le competenze multidisciplinari e transcalari di ogni attore del processo (tra cui l'organo statale), né appunto la formazione di una cultura politica limpida e consapevole dei ruoli e pesi che gravano sull'intero territorio nazionale, gli sforzi profusi rischiano di non essere sufficienti ad affrontare il problema della "casa".

L'inserimento abitativo (al pari di quello lavorativo) dei rifugiati rappresenta, infatti, una sfida troppo ardua per i soli attori locali. Il ripensamento del sistema di integrazione abitativa ha bisogno di uno sforzo congiunto di una filiera istituzionale centrale-regionale-locale, di obiettivi condivisi e strategie di lungo respiro, per interrompere l'attuale clima di incertezza, emergenzialità, degrado e discriminazione. 


\section{Riferimenti bibliografici}

Ministero dell'Interno (2018). Manuale sulla autonomia abitativa dei rifugiati. Milano, FarsiProssimo. https:// www.franoi.org/images/FR_materiali/Manuale\%20autonomia\%20abitativa.pdf

Comune di Milano (2018). Rapporto statistico sui rifugiati e $i$ Richiedenti Asilo a Milano: analisi delle fonti e dei dati. IX rapporto (dati 2018). Milano. https:// www.comune.milano.it/documents/20126/1002052/ Report+Rifugiati+dati+2018.pdf/1f5fb 1bb-ba9e-4a99-6bba-22d5e79966d3?t=1609318207130

Adam, F., Föbker, S., Imani, D., Pfaffenbach, C., Weiss, G., Wiegandt, C.C. (2020). Municipal Housing Strategies for Refugees. Insights from Two Case Studies in Germany. In Glorius, B., Doomernik, J. (a cura di). Geographies of Asylum in Europe and the Role of European Localities. Cham, Springer, 201-224.

Agamben, G. (2005), Homo sacer. Il potere sovrano e la nuda vita. Einaudi, Torino.

Ager, A., Strang, A. (2008). Understanding integration: a conceptual framework. Journal of Refugee Studies, 21, 166-191.

Agustoni, A. (2018). Segregation, Public Housing and Migratory Issues. The Italian case. In Alietti, A., Agustoni, A. (a cura di). Housing Policies, Migrants and Integration. Roma, Aracne, 85-106.

Aigner, A. (2019). Housing entry pathways of refugees in Vienna, a city of social housing. Housing Studies, 34 (5), 779-803. doi: 10.1080/02673037.2018.1485882

Alexander, M. (2007). Cities and labour immigration: comparing policy responses in Amsterdam, Paris, Rome and Tel Aviv. Aldershot, Ashgate Publishing.

Allen, J., Barlow, J., Leal, J., Maloutas., T., Padovani, L. (2004). Housing and Welfare in Southern Europe, Oxford, Blackwell Publishing.

Amato, F. (2008). Atlante dell'immigrazione in Italia. Roma, Carocci.

Ambrosini, M. (2010). Richiesti e respinti. L'immigrazione in Italia. Come e perché. Milano, Il Saggiatore.

Anderson, R. (2016). Europe's failed 'fight' against irregular migration: ethnographic notes on a counterproductive industry. Journal of Ethnic and Migration Studies, 42 (7), 1055-1075. doi: 10.1080/1369183X.2016.1139446

Andreotti, A., Mingione, E. (2016). Local welfare systems in Europe and the economic crisis. Europe- an Urban and Regional Studies, 23 (3), 252-266. doi: 10.1177/0969776414557191

Aru, S. (2019). Spazi d'asilo. Il sistema di accoglienza in Italia tra norme e politiche alle diverse scale territoriali. Geotema, 61, 34-40.

Babels. (a cura di). (2018). Entre accueil et rejet: ce que les villes font aux migrants. Lyon, Le Passager Clandestin.

Berry, J. (2006). Mutual attitudes among immigrants and ethnocultural groups in Canada. International Journal of Intercultural Relations, 30, 719-734. doi: 10.1016/j.ijintrel.2006.06.004

Bini, V., Gambazza, G. (2019). The reception of asylum seekers in urban areas: the case of the city of Milan. Belgeo, (1), 1-16. doi: 10.4000/belgeo.35559

Bricocoli, M., Cucca, R. (2016). Social mix and housing policy: Local effects of a misleading rhetoric. The case of Milan. Urban Studies, 53 (1), 77-91. doi: $10.1177 / 0042098014560499$

Brusa, C. (a cura di). (2012). Immigrazione e processi di interazione culturale. Geotema, 43-44-45.

Caponio, T., Borkert, M. (2010). The local dimension of migration policymaking. IMISCOE reports. Amsterdam, Amsterdam University Press.

Caponio, T., Scholten. P., Zapata-Barrero, R. (2019). The Routledge handbook of the governnance of migration and diversity in cities. Oxon-New York, Routledge.

Caruso, N. (2017). Policies and practices in Italian Welfare Housing. Turin, up to the curent neo-liberal approach and social innovation practices. Cham, Springer.

Cassi, L. Meini, M. (a cura di). (2002), L'immigrazione in carte. Per unanalisi a scala regionale dell'Italia. Geotema, 16.

Castles, S., Korac, M., Vasta, E., Vertovec, S. (2002), Integration Mapping the Field, in Home Office Online Report 29/03. London, Home Office.

Cittalia. (2010). Comuni e la questione abitativa. Le nuove domande sociali, gli attori e gli strumenti operativi. Second Edition. http://www.anci.it/Contenuti/Allegati/ Questione\%20abitativa.pdf.

Clapham, D. (2002). Housing pathways: A postmodern analytical framework. Housing, Theory and Society, 19 (2), 57-68.

Collyer, M., King, R. (2016). Narrating Europe's migration and refugee 'crisis'. Human Geography: a new radical journal, 9 (2), 1-12. doi: 10.1177/194277861600900201 
Cristaldi, F. (2012). Immigrazione e territorio. Lo spazio con/diviso. Bologna, Pàtron Editore.

Cristaldi, F. (2019). Migrazioni e processi territoriali in Italia. Geotema, 61, 3-9

Darling Jonathan (2013), Moral urbanism, asylum and the politics of critique. Environment and Planning $A$, 45(8), 1785-1801. doi:10.1068/a45441

Darling, J. (2017). Forced migration and the city: Irregularity, informality, and the politics of presence. Progress in Human Geography, 41 (2), 178-198. doi: 10.1177/0309132516629004

Derrida, J. (2001). On cosmopolitanism and forgiveness. Londra, Routledge.

Diab, B., Paradiso, M., Schnell, I. (2019). Ethnic Minorities' Embeddedness in Host Versus Origin Places. In Paradiso, M. (a cura di). Mediterranean mobilities: Europe's Changing Relationships. Cham, Springer, 77-88.

Eurocities (2015). Statement on asylum in cities. http:// nws.eurocities.eu/MediaShell/media/Final\%20EUROCITIES\%20statement\%20on\%20asylum.pdf

European Migration Network-EMN. (2018). Rapporto su asilo e migrazione in Italia, 2018. https://ec.europa. eu/home-affairs/sites/homeaffairs/files/15a_italy_annual_ policy_report_part2_2018_en.pdf

Ferrario, C. (2019). Immigrazione e territorio a Novara: dall'accoglienza alla stabilizzazione abitativa. Geotema, 61, 116-125.

Gavinelli, D., Santini, A. (2013). Immigrati e paesaggio: gli ethnoscapes nella città di Novara. In Aru, S., Corsale, A., Tanca, M. (a cura di). Percorsi migratori della contemporaneità: forme, pratiche, territori. Cagliari, CUEC, 215224.

Gidron, N., Hall, P.A. (2020). Populism as a Problem of Social Integration. Comparative Political Studies, 53 (7), 1027-1059. doi: 10.1177/0010414019879947

Gill, N. (2010). New state-theoretic approaches to asylum and refugee geographies. Progress in Human Geography, 34 (5), 626-645. doi: 10.1177/0309132509354629

Giudici, C., Wihtol de Wenden, C. (2016). I nuovi movimenti migratori. Il diritto alla mobilità e le politiche di accoglienza. Milano, FrancoAngeli.

Glick Schiller, N., Çağlar, A. (2009). Towards a comparative theory of locality in migration studies: migrant incorporation and city scale. Journal of Ethnic and Migration Studies. 35 (2), 177-202. doi: 10.1080/13691830802586179
Glick-Schiller, N., Çağlar, A. (2011). Locality and globality. Building a comparative analytical framework in migration and urban studies. In Glick-Schiller, N., Çağlar, A. (a cura di). Locating migration. Rescaling cities and migrants. Londra, Cornell University Press, 60-81.

Glorius, B., Doomernik, J. (2020). Introduction. In Glorius, B., Doomernik, J. (a cura di). Geographies of Asylum in Europe and the Role of European Localities Route. Cham, Springer, 1-11.

Harvey, D. (1973). Social justice and the city. Londra, Arnold.

ISMU (2019). Ventiquattresimo rapporto sull'immigrazione. Milano, FrancoAngeli.

Krasna, F. (2009). Alla ricerca dell'identità perduta. Una panoramica degli studi geografici sull'immigrazione straniera in Italia. Bologna, Pàtron.

Kreichauf, R. (2018). From forced migration to forced arrival: the campization of refugee accommodation in European cities. Comparative Migration Studies, 6 (article 7), 1-22. doi: 10.1186/s40878-017-

Meini, M., Salvatori, F. (a cura di). (2018). Per una geopolitica delle migrazioni. Roma, Società Geografica Italiana ONLUS.

Naga (2019). Senza (s)campo. Lo smantellamento del sistema di accoglienza per richiedenti asilo e rifugiati. Un'indagine qualitativa. https://naga.it/wp-content/uploads/2019/12/Report_Senza-scampo_Naga-5.pdf

Netto, G. (2011). Identity negotiation, pathways to housing and "place": the experience of refugees in Glasgow. Housing, Theory and Society, 28 (2), 123-143. doi: $10.1080 / 14036096.2010 .503676$

Omenetto, S. (2019). La rete SPRAR/SIPROIMI nelle strategie di valorizzazione del territorio. Il caso delle Comunità Montane e delle Unioni Montane titolari di progetti di seconda accoglienza. Geotema, 61, 57-65.

Phillips, D. (2006). Moving Towards Integration: The Housing of Asylum Seekers and Refugees in Britain. Housing Studies, 21 (4), 539-553. doi: 10.1080/02673030600709074

Phillips, D., Robinson, D. (2015). Reflections on Migration, Community, and Place. Population Space Place, 21 (5), 409-420. doi: 10.1002/psp.1911

Pittini, A., Koessl, G., Dijol, J., Lakatos, E., Ghekiere, L. (a cura di). (2017). The state of housing in the EU 2017. Bruxelles, Housing Europe. 
Putnam, R. (2000). Bowling alone: The collapse and revival of American community. New York, Simon and Schuster.

Puja, V. (2018). Il nuovo welfare abitativo lombardo. Opportunità per chi? Roma, Sbilanciamoci. https://sbilanciamoci.info/modello-welfare-abitativo-lombardoopportunita/

Robinson, V., Andersson, R., Musterd, S. (2003). Spreading the 'burden'? A review of policies to disperse asylum seekers and refugees. Bristol University Press, Bristol.

Sanyal, R. (2012). Refugees and the City: An Urban Discussion. Geography Compass, 6 (11), 633-644. doi: 10.1111/gec3.12010

Scholten, P. (2019). Two worlds apart? Multilevel governance and the gap between national and local integration policies. In Caponio, T., Scholten, P., Zapata-Barrero, R. The Routledge handbook of the governnance of migration and diversity in cities. Oxon-New York, Routledge, 178188.

Scholten, P., Penninx, R. (2016). The multilevel governance of migration and integration. In Garcés-Mascareñas, B., Penninx, R. (a cura di). Integration Processes and Policies in Europe. Contexts, Levels and Actors. New YorkLondra, Springer, 91-108.

Semprebon, M., Pelacani, G. (2020). Dispersal and Reception in Northern Italy: Comparing Systems Along the Brenner. In Glorius, Birgit, Doomernik, J. (a cura di). Geographies of Asylum in Europe and the Role of European Localities Route. Cham, Springer, 15-44.

Terranova, G. (2017). Il XXI secolo dell'immigrazione. Roma, Edicusano.

United Nations High Commission for Refugees. (2009). UNHCR Policy on Refugee Protection and Solutions in Urban Areas. Geneva, UNHCR.

Wolffhardt, A., Conte, C., Huddleston, T. (2019). The European benchmark for refugee integration: a comparative analysis of the national integration evaluation mechanism in 14 EU countries. Varsavia, Institute of Public Affairs.

Young, J. (2011). 'A new politics of the city': Locating the limit of hospitality and practicing the city-as refuge. Acme, 10 (3), 534-563.

Zapata-Barrero, R., Caponio, T., Scholten, P. (2017). Theorizing the 'local turn' in a multi-level governance framework of analysis: a case study in immigrant policies. International Review of Administrative Sciences, 83 (2), 241-246. doi: 10.1177/0020852316688426 https://www.camera.it/application/xmanager/projects/ leg18/attachments/upload_file_doc_acquisiti/ pdfs/000/001/791/Memorie_Prefetto_Michele_Di_Bari. pdf 